\title{
An investigation of the coordination chemistry of the hexadentate ligand, di- 2-pyridylketone azine; the formation of a discrete tetranuclear complex with silver nitrate
}

\author{
Christopher J. Sumby and Peter J. Steel* \\ Department of Chemistry, University of Canterbury, Christchurch, New Zealand. \\ Email: p.steel@chem.canterbury.ac.nz

\begin{abstract}
This submission was created using the RSC Article Template (DO NOT DELETE THIS TEXT) (LINE INCLUDED FOR SPACING ONLY - DO NOT DELETE THIS TEXT)
\end{abstract}

The ligand di-2-pyridylketone azine (1) was prepared by reaction of di-2-pyridylketone with hydrazine hydrate. This potentially hexadentate compound incorporates two extra imine donor atoms over other, previously investigated, ligands derived from di-2pyridylmethane. The coordination chemistry of 1 was investigated with silver nitrate, copper nitrate and palladium chloride. Reaction of $\mathbf{1}$ with silver nitrate gave a discrete tetranuclear complex (4), while with copper nitrate decomposition of the original ligand gave copper complexes, (5) and (6), of the ligands 3-(2-pyridyl)-triazolo[1,5-a]pyridine (2) and di-2-pyridylmethanediol (3), respectively. The complexes were characterised by elemental analyses and X-ray crystallography.

\section{Introduction}

The controlled self-assembly of metallosupramolecular species from combinations of bridging organic ligands and transition metal precursors has been the focus of much attention in recent years. $^{1-4}$ By employing various organic ligands, containing multiple heterocyclic rings that act as bridges in the formation of multinuclear metal complexes, it is possible to effect the controlled assembly of diverse molecular architectures, such as two-dimensional polygons and three-dimensional polyhedra. ${ }^{1,5}$

Bridging ligands incorporating 2,2'-bipyridine (bpy) subunits coordinate to metal atoms with the formation of stable five-membered chelate rings and have been extensively studied in coordination chemistry and for the synthesis of metallosupramolecular species. ${ }^{1,6}$ Much less studied within this context is the class of ligands, shown in Scheme 1, that contain two 2-pyridyl substituents separated by a single atom spacer (X). Upon coordination to a metal atom these ligands form a six-

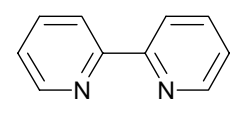

bpy

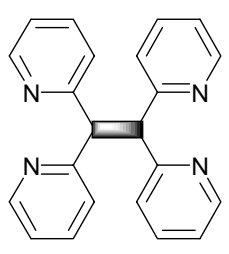

$\square=\mathrm{CH}-\mathrm{CH}$

$=\mathrm{C}(\mathrm{OH})-\mathrm{CH}$

$=\mathrm{C}=\mathrm{C}$

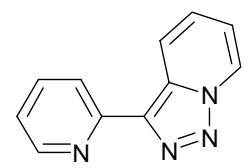

(2)

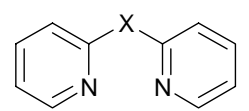

$\mathrm{X}=\mathrm{CH}_{2}(\mathrm{dpm})$

$=\mathrm{C}=\mathrm{O}(\mathrm{dpk})$

$=\mathrm{NH}, \mathrm{O}, \mathrm{S}$

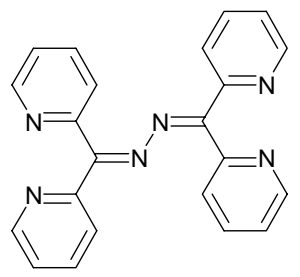

(1) membered chelate ring. ${ }^{7}$ The simplest of these is that in which a methylene group ( $\left.\mathrm{X}=\mathrm{CH}_{2}\right)$ acts as the spacer. Di-2pyridylmethane (dpm) has been sparingly used as a ligand, ${ }^{8}$ whereas di-2-pyridylketone (dpk, $\mathrm{X}=\mathrm{C}=\mathrm{O}$ ) has been more thoroughly investigated. ${ }^{9}$ We have recently reported investigations into the coordination and metallosupramolecular chemistry of a series of bridging ligands containing di-2pyridylmethane subunits (Scheme 1). ${ }^{3,4,10,11}$ In this paper we report an extension of that work with a study of the coordination chemistry of di-2-pyridylketone azine (1). Part of our interest in this ligand is that it provides a systematic increase in the metalmetal distances over previous ligands investigated by us, whilst still maintaining conjugation throughout the system. It also incorporates two further imine donor atoms over previously studied ligands to provide a potentially hexadentate ligand.

Although 1 has previously been prepared by acid-catalysed condensation of di-2-pyridylketone with hydrazine, ${ }^{12}$ an investigation of its coordination chemistry has not been undertaken. A survey of the Cambridge Structural Database ${ }^{13}$ (CSD version 5.24, July 2003) revealed there are no reports of complexes involving ligand $\mathbf{1}$ that have been characterised by Xray crystallography. Compound $\mathbf{1}$ has previously been investigated as a potential reagent for the spectrometric detection of various transition metals in biological water samples. ${ }^{12,14}$ Rich coordination chemistry has been reported for related heterocyclic ligands incorporating an azine backbone, which further prompted our studies of $\mathbf{1}$.

Included in this paper is an investigation of the coordination chemistry of $\mathbf{1}$ with silver(I), copper(II) and palladium(II) metal salts. The first crystal structure of a complex of $\mathbf{1}$ is described, along with the copper-catalysed decomposition of $\mathbf{1}$. The resulting decomposition products were characterised by $\mathrm{X}$-ray crystallography and revealed the formation of ligands 3-(2pyridyl)-triazolo[1,5-a]pyridine (2) and di-2-pyridylmethanediol (3) (Scheme 1).

\section{Experimental}

\section{General Experimental}

Di-2-pyridylketone (dpk) was purchased from Aldrich and used as received. NMR spectra were recorded on a Varian $500 \mathrm{MHz}$ NMR spectrometer. Melting points were performed on an Electrothermal melting point apparatus and are uncorrected. Elemental analyses were performed by the Campbell microanalytical laboratory at the University of Otago.

Scheme 1. 


\section{Preparations}

Synthesis of di-2-pyridylketone azine (1)

Di-2-pyridylketone azine was prepared by a modification of the method of Grases et al. ${ }^{12}$ Di-2-pyridylketone (203 mg, 1.10 $\mathrm{mmol})$, hydrazine hydrate $(26 \mu \mathrm{L}, 0.53 \mathrm{mmol})$ and acetic acid (3 drops) were refluxed overnight in methanol $(4 \mathrm{~mL})$. The methanol was removed in vacuo and the yellow solid, 1, was collected, washed with several portions of ether and dried. Yield 172 mg (89\%). M.p. $176-179^{\circ} \mathrm{C}$. Analysis: calc. for $\mathrm{C}_{22} \mathrm{H}_{16} \mathrm{~N}_{6} \mathrm{C}$ 72.51, H 4.43, N 23.06; found C 72.51, H 4.45, N 23.00\%. ${ }^{1} \mathrm{H}$ NMR $\left(\mathrm{CDCl}_{3}\right) \delta 8.70$ (d, 2H, H6'), 8.55 (d, 2H, H6”), 7.87 (d, 2H, H3”), 7.78 (t, 2H, H4'), 7.70 (m, 4H, H4”, H3'), 7.30 (dd, 2H, H5'), 7.27 (dd, 2H, H5”). ${ }^{13} \mathrm{C}$ NMR $\left(\mathrm{CDCl}_{3}\right) \delta 157.0,155.0$, 153.2, 149.1, 148.9, 136.4, 135.9, 125.6, 124.0, 123.6, 123.1. ${ }^{1} \mathrm{H}$ NMR ( $\left.\mathrm{CD}_{3} \mathrm{CN}\right) \delta 8.60$ (d, 2H, H6'), 8.44 (d, 2H, H6”), 7.80 (m, 6H, H3', H4', H4”), 7.64 (d, 2H, H3”), 7.32 (m, 4H, H5', H5”). ${ }^{13} \mathrm{C}$ NMR $\left(\mathrm{CD}_{3} \mathrm{CN}\right) \delta 158.9,156.4,155.1,150.1,149.9,137.7$, 136.7, 126.4, 125.4, 124.7, 123.6.

\section{Complexes of 1}

[ $\left.\mathbf{A g}_{\mathbf{4}}(\mathbf{1})_{\mathbf{2}}\left(\mathbf{N O}_{3}\right)_{\mathbf{4}}\right]$ (4). Ligand $\mathbf{1}(20.6 \mathrm{mg}, 0.056 \mathrm{mmol})$ was dissolved in methanol and reacted with silver nitrate $(20.2 \mathrm{mg}$, $0.112 \mathrm{mmol}$ ) also dissolved in methanol. Slow evaporation of the resulting solution gave a yellow crystalline precipitate, which contained yellow crystals suitable for X-ray crystallography. Yield $20.3 \mathrm{mg}$ (51\%). M.p. $235^{\circ} \mathrm{C}$. ${ }^{1} \mathrm{H}$ NMR $\left(\mathrm{CD}_{3} \mathrm{CN}\right) \delta 8.42$ (d, 2H, H6'), 8.38 (d, 2H, H6”), 7.90 (m, 4H, H4', H4”), 7.60 (m, 6H, H3', H5', H5”), 7.28 (d, 2H, H3”). ${ }^{13} \mathrm{C}$ NMR $\left(\mathrm{CD}_{3} \mathrm{CN}\right) \delta$ $163.4,152.7,151.8,151.3,150.5,146.3,140.1,139.0,129.1$, 128.6, 127.2. Analysis: calc. for $\mathrm{C}_{22} \mathrm{H}_{16} \mathrm{~N}_{8} \mathrm{O}_{6} \mathrm{Ag}_{2} \mathrm{C} 37.53, \mathrm{H}$ 2.29, N 15.91; found C 37.09, H 2.31, N 15.72\%.

$\left[\mathrm{Cu}(2)_{2}\left(\mathrm{NO}_{3}\right)_{2}\right]$ (5) and $\left[\mathrm{Cu}(3)_{2}\right]\left(\mathrm{NO}_{3}\right)_{2} \cdot \mathrm{H}_{2} \mathrm{O}$ (6). Copper nitrate $(27.7 \mathrm{mg}, 0.115 \mathrm{mmol})$ and $\mathbf{1}(20.5 \mathrm{mg}, 0.056 \mathrm{mmol})$ were both dissolved in methanol and the solutions mixed together. Slow evaporation initially gave green crystals of $\mathbf{5}$, followed by blue crystals of $\mathbf{6}$, when the solution was evaporated to dryness. Both sets of crystals were suitable for X-ray crystallography. Complex 5: Yield $8.3 \mathrm{mg}$ (51\%). M.p. $257^{\circ} \mathrm{C}$ (dec.). Analysis: calc. for $\mathrm{C}_{22} \mathrm{H}_{18} \mathrm{~N}_{10} \mathrm{O}_{6} \mathrm{Cu} \mathrm{C} 45.56, \mathrm{H} 2.78, \mathrm{~N}$ 24.15; found C 45.79, H 2.76, N 23.74\%. Complex 6: Yield 4.6 mg (26\%). M.p. $254-256^{\circ} \mathrm{C}$ (dec.). Complex 6 is identical to a previously reported copper complex formed by in situ decomposition of the ligand 1,1,2,2-tetrakis(2-pyridyl)ethan-1-ol following reaction with copper nitrate. ${ }^{11}$

Attempted preparations of $\left[\mathrm{Pd}_{\mathbf{2}}(\mathbf{1}) \mathrm{Cl}_{\mathbf{4}}\right]$ (7). (a) Palladium chloride $(21.3 \mathrm{mg}, 0.120 \mathrm{mmol})$ was dissolved in $2 \mathrm{M}$ hydrochloric acid $(5 \mathrm{~mL})$ and added slowly to a hot methanolic solution of 1 (20.1 mg, $0.055 \mathrm{mmol}$ ). The solution turned orange and a red-orange solid precipitated immediately. This was collected by filtration and dried in vacuo. Yield $21.0 \mathrm{mg} .{ }^{1} \mathrm{H}$ NMR showed a mixture of compounds to be present.

(b) Sodium tetrachloropalladate $(83.5 \mathrm{mg}, 0.284 \mathrm{mmol})$ and 1 (49.8 mg, $0.137 \mathrm{mmol}$ ) were both dissolved in methanol and mixed. A fluffy yellow solid precipitated from an orange solution. The yellow solid was collected by filtration, washed with methanol and ether, and dried under suction. Yield $48.3 \mathrm{mg}$. As above, ${ }^{1} \mathrm{H}$ NMR indicated a mixture of compounds to be present. Analysis: calc. for $\mathrm{C}_{22} \mathrm{H}_{16} \mathrm{~N}_{6} \mathrm{Cl}_{4} \mathrm{Pd}_{2} \cdot \mathrm{H}_{2} \mathrm{O} \mathrm{C} 35.85, \mathrm{H}$ 2.47, N 11.40; found C 35.65, H 1.60, N 10.55\%.

\section{Crystallography}

The crystal data, data collection and refinement parameters are listed below. Measurements were made with a Siemens CCD area detector using graphite monochromatised Mo $\mathrm{K} \alpha(\lambda=$ $0.71073 \AA$ ) radiation. The intensities were corrected for Lorentz and polarisation effects and for absorption. ${ }^{15}$ The structures were solved by direct methods using SHELXS, ${ }^{16}$ and refined on $F^{2}$ using all data by full-matrix least-squares procedures using SHELXL-97. ${ }^{17}$ All non-hydrogen atoms were refined with anisotropic displacement parameters. Hydrogen atoms were included in calculated positions with isotopic displacement parameters 1.2 times the isotropic equivalent of their carrier carbon atoms. The hydrogen atoms on the solvate water molecules in compound $\mathbf{4}$ were located in difference maps and refined with AFIX 3 constraints on the $\mathrm{O}-\mathrm{H}$ bonds. One nitrate anion (N135, O13, O14, O15, part 1; N140, O13', O14', O15', part 2) is disordered over two positions and these groups were restrained to a standard geometry.

Crystallographic data, as CIF files, have been deposited with the Cambridge Crystallographic Data Centre (CCDC No 228063 and 228064). Copies can be obtained free of charge from: The Director, CCDC, 12 Union Road, Cambridge CB2 1EZ, U.K. (email: deposit@ccdc.cam.ac.uk).

Crystal data for 4: $\mathrm{C}_{88} \mathrm{H}_{86} \mathrm{Ag}_{8} \mathrm{~N}_{32} \mathrm{O}_{35}, M=3014.85$, monoclinic, $a=13.585(1), b=16.163(2), c=48.468(5) \AA, \beta=95.559(1)^{\circ}, V$ $=10593(2) \AA^{3}, T=173 \mathrm{~K}$, space group $P 2_{1} / \mathrm{c}, Z=4$, yellow block, $0.64 \times 0.35 \times 0.11 \mathrm{~mm}^{3}, \mu=1.545 \mathrm{~mm}^{-1}, 131824$ reflections measured, 21434 unique $\left[R_{\text {int }}=0.0887\right], R_{1}[\mathrm{I}>2 \sigma(\mathrm{I})]$ $=0.0487, w R_{2}$ (all data) $=0.0990$.

Crystal data for 5: $\mathrm{C}_{22} \mathrm{H}_{16} \mathrm{CuN}_{10} \mathrm{O}_{6}, M=$ 579.99, triclinic, $a=7.030(3), b=9.039(4), c=9.806(4) \AA, \alpha=76.783(6)$, $\beta=75.962(6), \gamma=68.600(6)^{\circ}, V=556.0(4) \AA^{3}, T=173 \mathrm{~K}$, space group $P-1, Z=1$, green plate, $0.37 \times 0.16 \times 0.05 \mathrm{~mm}^{3}, \mu=1.049$ $\mathrm{mm}^{-1}, 7252$ reflections measured, 2235 unique $\left[R_{\mathrm{int}}=0.0223\right], R_{1}$ $[\mathrm{I}>2 \sigma(\mathrm{I})]=0.0295, w R_{2}($ all data $)=0.0678$.

\section{Results}

\section{Synthesis}

Di-2-pyridylketone azine (1) was prepared by reaction of di-2pyridylketone with hydrazine hydrate in the presence of a catalytic quantity of acetic acid. This provided the ligand, 1, as a yellow solid in $89 \%$ yield. The ligand was characterised by elemental analysis and NMR spectroscopy. While $\mathbf{1}$ has the same di-2-pyridylmethyl coordination motif as ligands we have previously investigated, ${ }^{10,11}$ it also possesses an azine backbone that provides two additional imine nitrogen donor atoms, producing a potentially hexadentate ligand. Complexes of $\mathbf{1}$ were prepared by reactions with silver nitrate, copper nitrate and palladium chloride (Scheme 2) to assess the involvement of the six potential donors in coordination complexes of this ligand.

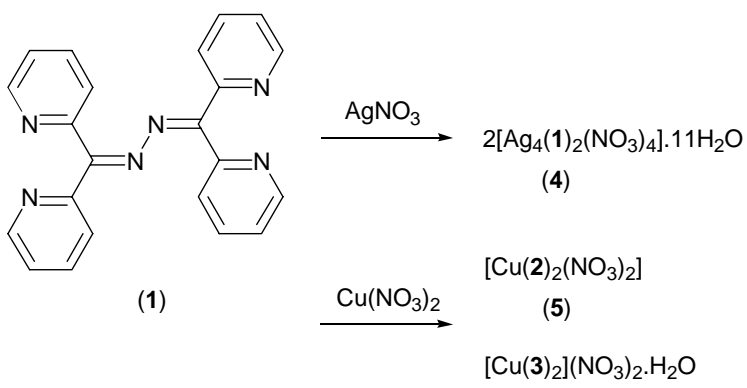

(6)

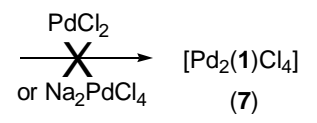

Scheme 2. 
Ligand 1 was reacted with silver nitrate in a 1:2 ratio to give a yellow crystalline complex (4) in 51\% yield. Elemental analysis revealed the product had the expected 2:1 metal-ligand ratio, suggesting the possibility of a discrete $\mathrm{M}_{2} \mathrm{~L}$ complex. Fortunately, crystals suitable for X-ray crystallography were subsequently obtained on slow evaporation of the methanol reaction mixture. X-Ray structure determination confirmed that the silver complex was indeed discrete, but that it had an unusual tetranuclear formulation, $\left[\mathrm{Ag}_{4}(\mathbf{1})_{2}\left(\mathrm{NO}_{3}\right)_{4}\right] .{ }^{1} \mathrm{H}$ NMR studies on complex $\mathbf{4}$ showed that the symmetry of the ligand is maintained in solution. Changes in the chemical shifts, consistent with coordination to silver and the formation of the tetranuclear structure 4, are observed. In particular, the chemical shifts for the H6 protons of the ligand in complex 4 move up-field (8.60 and $8.44 \mathrm{ppm}$ for 1 ; 8.42 and $8.38 \mathrm{ppm}$ for 4 ), consistent with the Xray structure in which these hydrogens lie over the shielding regions of adjacent pyridine rings.

Reaction of $\mathbf{1}$ with copper nitrate has previously been investigated by Grases et al. during evaluation of $\mathbf{1}$ as a potential spectrofluorometric reagent for determination of copper. ${ }^{12}$ In our hands reaction of $\mathbf{1}$ with copper nitrate yielded two different reaction products, corresponding to complexes of decomposition products ( 2 and 3 ) of the original ligand. Cleavage of one of the hydrazone units and cyclisation to give a triazolo[1,5- $a$ ]pyridine ring system gave complex $\left[\mathrm{Cu}(2)_{2}\left(\mathrm{NO}_{3}\right)_{2}\right](5)$. Complex 5 was isolated as green crystals in 51\% yield by slow, but incomplete evaporation of a methanol reaction mixture. These crystals were characterised by X-ray crystallography and a brief description of the structure, which closely resembles a previously reported copper complex ${ }^{18}$ is given below. Further evaporation of the reaction medium yielded blue crystals in $26 \%$ yield that were shown to be identical to a previously characterised copper complex, $\left[\mathrm{Cu}(3)_{2}\right]\left(\mathrm{NO}_{3}\right)_{2} \cdot \mathrm{H}_{2} \mathrm{O}(\mathbf{6}){ }^{11}$ These two products result from hydrolysis and oxidation of the initial azine ligand in the presence of copper.

We sought to prepare a dinuclear palladium complex of the azine ligand $\mathbf{1}$ by reaction of a solution of palladium chloride with a methanol solution of the ligand. The complex that formed as a precipitate from the reaction mixture directly upon mixing the two solutions, was a red solid. This analysed with a composition consistent with the anticipated dinuclear complex (7), but ${ }^{1} \mathrm{H}$ NMR indicated this solid to be a mixture of compounds, possibly including palladium complexes of compounds $\mathbf{2}$ and $\mathbf{3}$ resulting from decomposition of ligand $\mathbf{1}$. It is likely that the acidic conditions of the reaction promote such decomposition reactions. In an attempt to overcome this problem, the reaction was carried out in methanol using sodium tetrachloropalladate as a source of $\mathrm{PdCl}_{2}$. A yellow precipitate was obtained from this reaction, which again analyses close to the values expected for 7 , but was shown to be a mixture of compounds by ${ }^{1} \mathrm{H}$ NMR. Investigations are currently in progress to determine the exact composition of this material.

\section{Crystal structure of 4}

The structure of complex $\mathbf{4}$ was shown by X-ray crystallography to be a discrete $\mathrm{Ag}_{4} \mathrm{~L}_{2}$ tetranuclear complex, formed from the self-assembly of four silver atoms and two molecules of $\mathbf{1}$. The complex crystallises in the monoclinic space group $\mathrm{P} 2{ }_{1} / \mathrm{c}$, with an asymmetric unit that contains two complete units of the discrete complex (one of which is shown in Fig. 1), four non-coordinated nitrate anions and eleven water solvate molecules. In the perspective view shown in Fig. 1 one molecule of $\mathbf{1}$ has the bonds filled in black, while the second has hollow bonds. The other unit of the discrete complex has the same structure, but the chelating nitrate anion coordinated to $\operatorname{Ag}(4)$ (equivalent of $\mathrm{Ag}(8)$ in the pictured complex) is disordered over two positions.

Focusing on one of the discrete $\left[\mathrm{Ag}_{4}(\mathbf{1})_{2}\left(\mathrm{NO}_{3}\right)_{2}\right]$ units reveals that the four coordinated silver atoms lie in a plane between the two bridging ligands. Each hexadentate ligand utilises all six donors in bonding to the four silver atoms, although in the case

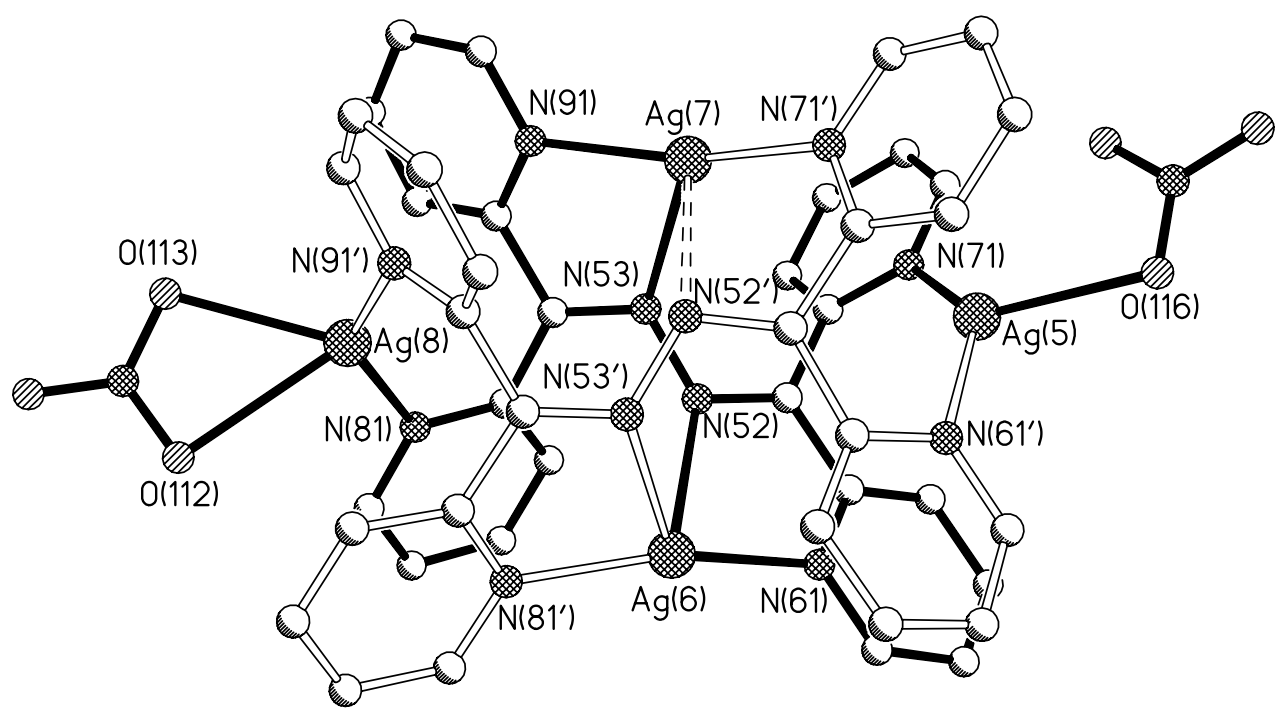

Fig. 1. A perspective view of one discrete complex of structure 4 with hydrogen atoms, non-coordinated anions and solvate water molecules omitted for clarity. Selected bond lengths ( $\AA$ ) and angles ( $\left.{ }^{\circ}\right)$ : $\mathrm{Ag}(5)-\mathrm{N}(71) 2.226(5), \mathrm{Ag}(5)-\mathrm{N}(61 ') 2.268(4), \mathrm{Ag}(5)-\mathrm{O}(116) 2.469(4), \mathrm{Ag}(6)-\mathrm{N}(61) 2.222(5), \mathrm{Ag}(6)-$ $\mathrm{N}\left(81^{\prime}\right)$ 2.229(4), $\mathrm{Ag}(6)-\mathrm{N}\left(53^{\prime}\right)$ 2.489(4), Ag(6)-N(52) 2.579(4), Ag(7)-N(71') 2.247(5), Ag(7)-N(91) 2.286(5), Ag(7)-N(53) 2.487(4), Ag(8)-N(91') 2.214(5), Ag(8)-N(81) 2.239(5), Ag(8)-O(113) 2.459(5), Ag(8)-O(112) 2.606(5), N(71)-Ag(5)-N(61') 140.3(2), N(71)-Ag(5)-O(116) 128.5(2), N(61')Ag(5)-O(116) 90.8(2), N(61)-Ag(6)-N(81') 164.8(2), N(61)-Ag(6)-N(53') 124.7(2), N(81')-Ag(6)-N(53') 70.5(2), N(61)-Ag(6)-N(52) 69.3(2), N(81')Ag(6)-N(52) 116.3(2), N(53')-Ag(6)-N(52) 83.3(1), N(71')-Ag(7)-N(91) 162.0(2), N(71')-Ag(7)-N(53) 126.3(2), N(91)-Ag(7)-N(53) 69.3(2), N(91')Ag(8)-N(81) 141.0(2), N(91')-Ag(8)-O(113) 106.3(2), N(81)-Ag(8)-O(113) 112.3(2), N(91')-Ag(8)-O(112) 117.4(2), N(81)-Ag(8)-O(112) 92.6(2), $\mathrm{O}(113)-\mathrm{Ag}(8)-\mathrm{O}(112) 50.1(1)$. 
of the pictured structure one of the imine nitrogen donors, $\mathrm{N}\left(52^{\prime}\right.$ '), makes a very weak contact (2.824(4) $\AA$ ) with $\mathrm{Ag}(7)$.

In each discrete complex the ligand caps four silver atoms, which all lie in a central plane. Recently, we described the first example of an $\mathrm{M}_{6} \mathrm{~L}_{2}$ structure belonging to a family of cage-like structures of formula $\mathrm{M}_{\mathrm{n}} \mathrm{L}_{2}{ }^{3}$. These compounds (Scheme 3) are comprised of two multipodal ligands bridging $n$ metal centres. Fujita $^{19}$ first described the formation of a trigonal structure (a) with three palladium atoms bridged by two tripodal ligands. The first tetragonal structure (b) employed symmetrical cavitands as bridges, ${ }^{20}$ and a novel organometallic example has been reported that used a cyclobutadienyl core. ${ }^{21}$ The tetranuclear structure described here is complicated by the presence of two additional donors and is better represented as (c).

Like the hexanuclear structure described by us previously, ${ }^{3}$ the tetranuclear system described here displays cooperative interactions between the pyridine rings. When viewed from above (as in Fig. 1), all the pyridine donors in the top ligand are twisted in an anticlockwise direction, while the pyridine nitrogen donors in the bottom ligand are twisted in a clockwise direction. Therefore each discrete complex is helical and chiral, although the overall crystal is racemic.

There are two different silver coordination environments in the structure; one where the silver is coordinated by pyridine donors and nitrate oxygen atoms (Ag(5) and $\mathrm{Ag}(8))$ and a second where the imine nitrogen atoms coordinate to the silver atoms in place of the nitrate donors $(\mathrm{Ag}(6)$ and $\mathrm{Ag}(7))$. $\mathrm{Ag}(5)$ and $\mathrm{Ag}(8)$ are coordinated by two pyridine nitrogen atoms and a weakly bonded nitrate anion. The pyridine $\mathrm{N}-\mathrm{Ag}$ bond lengths are in the range 2.214(5) to 2.286(5) $\AA$, while the closest Ag-O distances are 2.469(4) and 2.459(5) for $\operatorname{Ag}(5)$ and $\operatorname{Ag}(8)$, respectively. $\operatorname{Ag}(6)$ and $\operatorname{Ag}(7)$ both have approximate five-coordinate environments, with coordination by two pyridine nitrogen atoms and three other donors. $\operatorname{Ag}(6)$ is coordinated by the two imine nitrogen atoms of the azine, with distances of 2.489(4) and 2.579(4) $\AA$, and also makes a weak contact with a noncoordinated nitrate atom with a Ag-O distance of 2.895(5) $\AA$. $\mathrm{Ag}(7)$ makes one bond to an imine nitrogen atom (2.487(4) $\AA$ ) and a weak bond to a nitrate anion (2.616(4) $\AA$ ) in addition to a weak interaction with the other imine nitrogen as described above. There is a wide variance in the silver-silver distances within the complex, but none that could be considered even to be a weak bonding interaction. ${ }^{22}$ The shortest silver-silver distance $(\mathrm{Ag}(5)-\mathrm{Ag}(7))$ is 4.200(1) $\AA$, while $\mathrm{Ag}(5)$ and $\mathrm{Ag}(8)$ are separated by $8.048(1) \AA$.

Helical $\mathrm{Ag}_{2} \mathrm{~L}_{2}$ and $\mathrm{Ag}_{2} \mathrm{~L}_{3}$ complexes with a related ligand have recently been described by other workers. ${ }^{23,24}$ When 2acetylpyridine azine was reacted with $\mathrm{AgX}$ salts $(\mathrm{X}=$ perchlorate, tetrafluoroborate, hexafluorophosphate and nitrate), $\left[\mathrm{Ag}_{2}(\mathrm{~L})_{2}\right](\mathrm{X})_{2}$ and $\left[\mathrm{Ag}_{2}(\mathrm{~L})_{3}\right](\mathrm{X})_{2}$ complexes were obtained and characterised by $\mathrm{X}$-ray crystallography. These complexes are helicates, with the ligand providing the helical part of the complex, in contrast to the structure described in this paper,

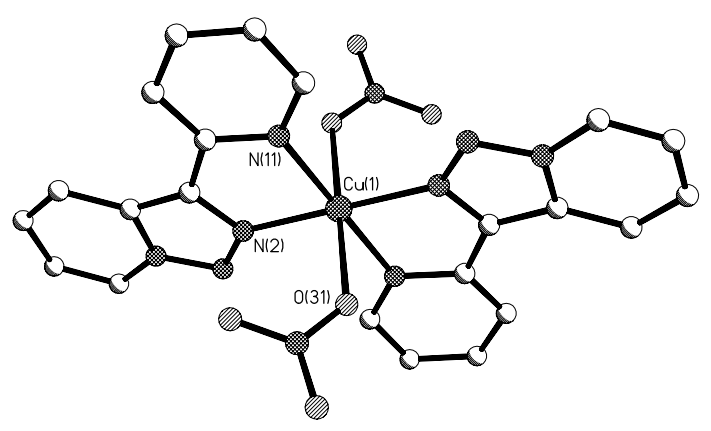

Fig. 2. A perspective view of complex 5. Selected bond lengths $(\AA)$ and angles ( $\left.{ }^{\circ}\right)$ : $\mathrm{Cu}(1)-\mathrm{N}(2)$ 2.0135(17), $\mathrm{Cu}(1)-\mathrm{N}(11) 2.0517(18), \mathrm{Cu}(1)-\mathrm{O}(31)$ 2.4324(18), N(2)-Cu(1)-N(11) 80.03(7), N(2)-Cu(1)-O(31) 97.91(7), $\mathrm{N}(11)-\mathrm{Cu}(1)-\mathrm{O}(31) 95.53(7)$.

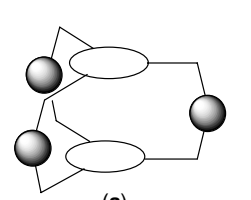

(a)

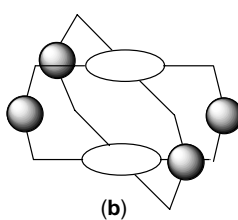

Scheme 3.

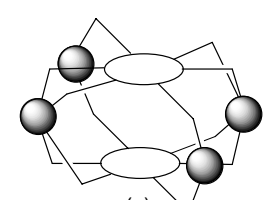

(c) where the silver atoms form the 'strands'. Triple stranded helicates have also been described with other transition metals. $^{23,25}$

\section{Crystal structure of 5}

The crystal structure of complex $\mathbf{5}$ confirms that ligand $\mathbf{1}$ has undergone a copper-catalysed decomposition to form 3-(2pyridyl)triazolo[1,5-a]pyridine (2). This ligand has previously been directly prepared and a copper nitrate complex, with almost an identical structure, characterised by X-ray crystallography. ${ }^{18}$ A similar decomposition has been noted for the closely related ligand, phenyl 2-pyridylketone azine on reaction with copper chloride. ${ }^{26}$ Ligand 2 was previously prepared by refluxing di-2pyridylketone and hydrazine hydrate in water-methanol solution. ${ }^{18}$ If the reaction was carried out in the absence of oxygen, ligand 2 was not obtained. It has also been prepared by manganese dioxide oxidation of the intermediate hydrazone ${ }^{27,28}$ and some iron complexes have been reported recently. ${ }^{28}$

The copper nitrate complex reported by Battaglia crystallises in the monoclinic space group $P 2_{1} / \mathrm{c}$ with two complete cations in the asymmetric unit. The copper atoms have a Jahn-Teller distorted octahedral geometry with water molecules as the apical ligands. By contrast the complex reported here crystallises in the triclinic space group $P-1(Z=1)$, with the copper atom on a centre of inversion. No water solvate molecules are present in this structure. A perspective view of the complex without hydrogen atoms is shown in Fig. 2. The bond lengths and angles are similar to the previously reported ${ }^{18}$ structures and are typical for a Jahn-Teller distorted copper complex. The bond distances to the pyridine nitrogen donor is $2.0517(18) \AA$, while the bond length for the copper-triazolo[1,5-a]pyridine bond is 2.0135(17) $\AA$. The nitrate anions are weakly bound, with copper-oxygen bond lengths of 2.4324(18) $\AA$.

Complex 5 also has a very similar structure to copper complexes reported by Richardson with benzisoxazole and benzotriazole-based ligands..$^{29,30}$ Unlike the species described here, one of the copper complexes reported by Richardson ${ }^{29}$ and the compound described by Battaglia et al., ${ }^{18}$ have the nitrate anions hydrogen bonded to axially coordinated solvate molecules. The third related structure, the copper nitrate complex of 3-(2-pyridyl)-1,2-benzisoazole ${ }^{30}$ has the nitrate anions directly coordinated to the copper centre. This latter complex has identical cell dimensions to complex $\mathbf{5}$ and thus the two structures are isomorphous, but not isostructural.

\section{Discussion}

The azine-based ligand $\mathbf{1}$ was easily and conveniently prepared by condensation of di-2-pyridylketone and hydrazine hydrate, two commercially available starting materials, in a single reaction. Other modifications can be envisioned which would allow the synthesis of a large range of related ligands. Some of these have been prepared and have, on reaction with various transition metal precursors, resulted in the formation of some interesting metallosupramolecular structures. ${ }^{23-25}$ Our study of the coordination chemistry of $\mathbf{1}$ revealed that the stability of such ligands may be a matter of concern, as evidenced in the decomposition of $\mathbf{1}$ in the presence of copper.

Reaction of $\mathbf{1}$ with silver nitrate led to the formation of an unusual tetranuclear assembly comprised of two molecules of the ligand and four silver atoms. This required the use of all four 
pyridine donors and the two azine nitrogen donor atoms of each ligand. Thus $\mathbf{1}$ has been shown to act as a hexadentate donor. Other potential hexadentate modes of coordination are possible in which the ligand could act as a doubly tridentate donor but this was not observed during the present work.

In the presence of copper, the azine bridge in $\mathbf{1}$ was shown to be susceptible to cleavage of the hydrazone units despite the extended delocalisation throughout the ligand system. Once hydrolysed the intermediate is subject to oxidation giving the triazolo[1,5- $a$ ]pyridine ring system. This may also be the case for the reactions involving palladium. Similar problems have not been reported with other azine ligands.

In summary, the coordination chemistry of this interesting hexadentate ligand has been investigated with three different transition metals and the results suggest that a range of coordination modes may be possible for this ligand, in addition to the complexes described here.

\section{References}

1 For reviews, see: S. Leininger, B. Olenyuk, and P. J. Stang, Chem. Rev., 2000, 100, 853; B. J. Holliday and C. A. Mirkin, Angew. Chem., Int. Ed., 2001, 40, 2022; B. Moulton and M. J. Zaworotko, Chem. Rev., 2001, 101, 1629; G. F. Sweigers and T. J. Malefetse, Chem. Rev., 2000, 100, 3483; M. J. Zaworotko, Chem. Commun., 2001, 1; S. L. James, Chem. Soc. Rev, 2003, 32, 276; M. J. Hannon and L. J. Childs, Supramol. Chem., 2004, 16, 7; F. Würthner, C.-C. You and C. R. Saha-Möller, Chem. Soc. Rev, 2004, 33, 133; H. Hofmeier and U. S. Schubert, Chem. Soc. Rev., 2004, 33, 373;

2 C. M. Hartshorn and P. J. Steel, Chem. Commun., 1997, 541; D. A. McMorran and P. J. Steel, Angew. Chem., Int. Ed., 1998, 37, 3295; D. A. McMorran and P. J. Steel, Chem. Commun., 2002, 2120; P. J. Steel, Acc. Chem. Res., 2005, 38, 243.

P. J. Steel and C. J. Sumby, Chem. Commun., 2002, 322.

P. J. Steel and C. J. Sumby, Inorg. Chem. Commun., 2002, 5, 323.

G. F. Sweigers and T. J. Malefetse, Chem. Eur. J., 2001, 7, 3637.

V. Balzani, A. Juris, M. Venturi, S. Campagna and S. Serroni, Chem. Rev., 1996, 96, 759; C. Kaes, A. Katz and M. W. Hosseini, Chem. Rev., 2000, 100, 3553; A. L. Gavrilova and B. Bosnich, Chem. Rev., 2004, 104, 349; G. R. Newkome, A. N. Patri, E. Holder and U. S. Schubert, Eur. J. Org. Chem., 2004, 235; A. J. Downard, I. G. Phillips and P.J. Steel, Aust. J. Chem., 2004, 57, 865, and references therein.

$7 \quad$ E. C. Constable and P. J. Steel, Coord. Chem. Rev., 1989, 93, 205.

8 A. J. Canty and N. J. Minchin, Aust. J. Chem., 1986, 39, 1063; A. J. Canty, N. Chaichit, B. M. Gatehouse, E. E. George and G. Hayhurst, Inorg. Chem., 1981, 20, 2414; E. Spodine, J. Manzur, M. T. Garland, J. P. Fackler, Jr., R. J. Staples and B. Trzcinska-Bancroft, Inorg. Chim. Acta, 1993, 203, 73.

9 P. K. Byers, A. J. Canty, L. M. Engelhardt, J. M. Patrick and A. H. White, J. Chem. Soc., Dalton Trans., 1985, 981; A. J. Canty, P. R. Traill, B. W. Skelton and A. H. White, Inorg. Chim. Acta, 1997, 255, 117; G. S. Papaefstathiou and S. P. Perlepes, Comments Inorg. Chem., 2002, 23, 249; M.-L. Tong, S.-L. Zheng, J.-X. Shi, Y.-X. Tong, H.-K. Lee and X.-M. Chen, J. Chem. Soc., Dalton Trans., 2002, 1727; W. L. Huang, J. R. Lee, S. Y. Shi and C. Y. Tsai, Transition Met. Chem., 2003, 28, 381.

10 D. M. D'Alessandro, F. R. Keene, P. J. Steel and C. J. Sumby, Aust. J. Chem., 2003, 56, 657.

11 P. J. Steel and C. J. Sumby, Dalton Trans., 2003, 4505.

12 F. Grases, F. Garcia-Sanchez and M. Valcarcel, Anal. Chim. Acta, 1981, 125, 21.

13 F. H. Allen, S. A. Bellard, M. D. Brice, B. A. Cartwright, A. Doubleday, H. Higgs, T. Hummelink, B. G. Hummelink-Peters, O. Kennard, W. D. S. Motherwell, J. D. Rodgers and D. G. Watson, Acta Cryst., 1979, B35, 2331.

14 F. Grases, J. M. Estela, F. Garcia-Sanchez and M. Valcarcel, Analysis, 1981, 9, 66; F. Grases, C. Genestar and J. J. Gil, Microchem. J., 1985, 31, 44.

15 G. M. Sheldrick, SADABS, University of Göttingen, Germany, 1998.

16 G. M. Sheldrick, Acta Cryst., 1990, A46, 467.

17 G. M. Sheldrick, SHELXL-97, University of Göttingen, Germany, 1997.

18 L. P. Battaglia, M. Carcelli, F. Ferraro, L. Mavilla, C. Pelizzi and G. Pelizzi, J. Chem. Soc., Dalton Trans., 1994, 2651.
19 M. Fujita, S. Nagao and K. Ogura, J. Am. Chem. Soc., 1995, 117, 1649.

20 P. Jacopozzi and E. Dalcanale, Angew. Chem., Int. Ed., 1997, 36, 613.

21 S. C. Johannessen, R. G. Brisbois, J. P. Fischer, P. A. Greico, A. E. Counterman and D. E. Clemmer, J. Am. Chem. Soc, 2001, 123, 3818.

22 P. Pyykko, Chem. Rev., 1997, 97, 597.

23 J. Hamblin, A. Jackson, N. W. Alcock and M. J. Hannon, J. Chem. Soc., Dalton Trans., 2002, 1635; F. Tuna, J. Hamblin, A. Jackson, G. Clarkson, N. W. Alcock and M. J. Hannon, Dalton Trans., 2003, 2141; F. Tuna, G. Clarkson, N. W. Alcock and M. J. Hannon, Dalton Trans., 2003, 2149.

24 G. Dong, H. Cheng, D. Chun-Ying, Q. Chun-Qi and M. Qing-Jin, New J. Chem., 2002, 26, 796; G. Dong, P. Ke-Liang, D. Chun-Ying, Z. Yong-Gang and M. Qing-Jin, Chem. Lett., 2002, 10, 1014.

25 G. Dong, P. Ke-Liang, D. Chun-Ying, C. He and M. Qing-Jin, Inorg. Chem., 2002, 41, 5978.

26 E. Amadei, M. Carcelli, S. Ianelli, P. Cozzini, P. Pelagatti and C. Pelizzi, J. Chem. Soc., Dalton Trans., 1998, 1025.

27 B. Abarca, R. Ballesteros and M. Elmasnaouy, Tetrahedron, 1998 , 54, 15287; B. Abarca, R. Ballesteros and M. Chadlaoui, Tetrahedron, 2004, 60, 5785.

28 V. Niel, A. B. Gaspar, M. C. Munoz, B. Abarca, R. Ballesteros and J. A. Real, Inorg. Chem.,2003, 42, 4782.

29 C. Richardson and P. J. Steel, Dalton Trans., 2003, 992.

30 C. Richardson and P. J. Steel, Inorg. Chem. Commun., 2000, 3, 155. 


\section{Graphical Abstract}

An investigation of the coordination chemistry of the hexadentate ligand, di-2-pyridylketone azine; the formation of a discrete tetranuclear complex with silver nitrate

\section{Christopher J. Sumby and Peter J. Steel}

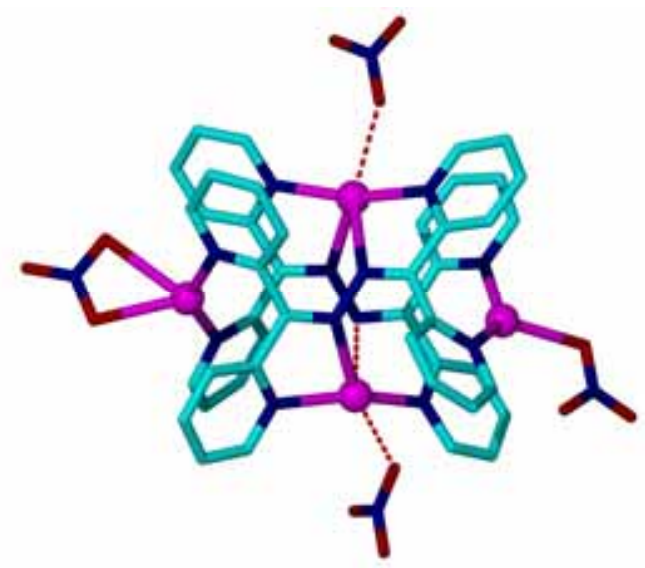

Reaction of the ligand di-2-pyridylketone azine with silver nitrate leads to the formation of a discrete $\mathrm{Ag}_{4} \mathrm{~L}_{2}\left(\mathrm{NO}_{3}\right)_{4}$ complex. 